\title{
Fabrication of AZO TCO Films by RF-sputtering and Their Physical Properties
}

\author{
T.S. Jang ${ }^{1}$, and D.C. $\mathrm{Oh}^{*}$ \\ ${ }^{1}$ Department of Nanobiotronics, Hoseo University, Sechul-ri, Baebang-eup, Asan-si, 31499, Korea
}

\begin{abstract}
We report on the fabrication of Al-doped $\mathrm{ZnO}$ (AZO) transparent-conductive oxide (TCO) films on glass substrates by RFsputtering, their physical properties, and the effect of thermal annealing on the AZO TCO films. The AZO films on glass substrates have a preferred orientation of the c-axis, irrespective of deposition conditions, which means that the AZO films have textured structures along the c-axis. The film thickness and surface roughness in the AZO films are proportional to plasma power and deposition time, while they are inverse-proportional to working gas ratio and working pressure. The AZO films have the optical transmittance over $80 \%$ in the wavelength range of $400-1000 \mathrm{~nm}$, irrespective of deposition conditions. The plasma power and the deposition time relatively give a large influence on the optical transmittance, compared to the working gas ratio and the working pressure. The AZO films deposited at room temperature have poor electrical properties, while the thermal annealing under $\mathrm{Ar}$ ambient significantly improves the electrical conductivity of the AZO films: an as-deposited sample has an electrical resistivity of $87 \mathrm{Wcm}$ and an electron concentration of $1.3^{\prime} 10^{17}$ $\mathrm{cm}^{-3}$, while the annealed sample has an electrical resistivity of $3.7^{\prime} 10^{-2}$ $\mathrm{Wcm}$ and an electron concentration of $1.2^{\prime} 10^{20} \mathrm{~cm}^{-3}$.
\end{abstract}

\section{Introduction}

Recently, transparent conductive oxide (TCO) has attracted strong attention as the unprecedented applications such as smart phone, tablet PC, and flat panel display, etc beyond the existing devices such as solar cell and LED come on stage one after another [13]. $\mathrm{AZO}, \mathrm{Al}$-doped $\mathrm{ZnO}$ has been studied as a promising material for transparent conductive oxides (TCOs) for a long time due to the wide bandgap of $3.37 \mathrm{eV}$ and the high n-type conductivity by native donor-type defects [1-3]. Thin film $\mathrm{ZnO}$ has the background electron concentration of high $10^{16}$ - high $10^{18} \mathrm{~cm}^{-3}$ due to native defects such as $\mathrm{H}, \mathrm{Zn}_{\mathrm{i}}$, and $\mathrm{V}_{\mathrm{O}}[4,5]$. The inappropriate fabrication conditions of the low growth temperature that is not enough to supply the formation energy of $\mathrm{ZnO}$ bonds and the heterosubstrate with a large lattice mismatch of $\mathrm{ZnO}$ unit cells, are known to generate the various donor-type native defects unintentionally, though the background electron concentration level of the $\mathrm{ZnO}$ films depends on their growth techniques [4,5]. Moreover, the n-type conductivity of

*Corresponding author: ohdongcheol@hoseo.edu 
$\mathrm{ZnO}$ can be effectively controlled by doping group III elements such as $\mathrm{Al}, \mathrm{Ga}$, and In. The electron concentration approaches to the level of low $10^{21} \mathrm{~cm}^{-3}$ and the electrical resistivity approaches to the level of low $10^{-4} \mathrm{Wcm}$ in the Al-doped $\mathrm{ZnO}$, Ga-doped $\mathrm{ZnO}$, and Indoped ZnO films, fabricated by pulsed-laser deposition (PLD), metal-ogranic chemicalvapor deposition (MOCVD), atomic-layer deposition (ALD), DC sputter, and RF sputter [6-10]. Especially, Al-doped $\mathrm{ZnO}$ is very powerful and effective when used in the target sources of sputters, because the sputtering system has the merits of low cost, massproductivity, and convenient maintenance, compared to other thin film fabrication systems such as PLD, MOCVD, and ALD. On the contrary, Ga-doped ZnO and In-doped ZnO have the difficulty that the volatile characteristics of high vapor pressure and low melting temperature in the $\mathrm{Ga}$ and In sources can cause the unexpected problems in the target fabrication and the sputtering process. Also, the AZO material has the merits of environment-friend and low-cost, compared to Indium-Tin-Oxide (ITO) that is widely used as a TCO material more than 100 years, because it does not have the rare-earth materials of In and $\mathrm{Sn}[1-3]$.

In this work, we report on the fabrication process of AZO TCO films on glass substrates by RF-sputtering, their physical properties such as surface, structural, optical, and electrical properties, and influence of thermal annealing on the electrical properties of AZO TCO films.

\section{Experimental details}

Four sets of AZO films as functions of plasma power, working gas ratio, working pressure, and deposition time were fabricated on glass substrates by using RF-sputter (SNTEK RSP5004) and AZO target of $\mathrm{ZnO}: \mathrm{Al}_{2} \mathrm{O}_{3}(=98: 2 \mathrm{wt} \%)$. First, the plasma power $\left(\mathrm{P}_{\text {plasma }}\right)$ is varied in the range of $50-200 \mathrm{~W}$ under the working gas ratios $\left(\mathrm{O}_{2} / \mathrm{Ar}+\mathrm{O}_{2}\right)$ of $0-100 \%$ and the working pressures $\left(\mathrm{P}_{\text {work }}\right)$ of $10-30 \mathrm{mT}$ for a deposition time $\left(\mathrm{t}_{\text {dep }}\right)$ of $30 \mathrm{~min}$. Second, the working gas ratio is varied in the range of $0-100 \%$ under the working pressures of $10-30 \mathrm{mT}$ at a plasma power of $100 \mathrm{~W}$ for a deposition time of $30 \mathrm{~min}$. Third, the deposition time is varied in the range of $5-60 \mathrm{~min}$ at a plasma power of $100 \mathrm{~W}$ under a working pressure of $10 \mathrm{mT}$ and a working gas ratio of 0 (Ar). Then substrate temperature is fixed at room temperature (RT). All fabricated samples were thermally annealed by using open-tube furnace at the temperature range of $200-500{ }^{\circ} \mathrm{C}$ under an $\mathrm{Ar}$ ambient for at a temperature of $60 \mathrm{~min}$.

Table 1. Fabrication conditions of AZO films and thermal annealing conditions.

\begin{tabular}{|c|c|}
\hline Target & ZnO (98 wt\%) $: \mathbf{A l}_{\mathbf{2}} \mathbf{O}_{\mathbf{3}}(\mathbf{2} \mathbf{~ w t} \%)$ \\
Substrate & $50,100,200$ \\
\hline Plasma power $\left(\mathrm{P}_{\text {plasma }}\right)(\mathrm{W})$ & $0(\mathrm{Ar}), 50,100\left(\mathrm{O}_{2}\right)$ \\
Working gas ratio $\left(\mathrm{O}_{2} / \mathrm{Ar}+\mathrm{O}_{2}\right)(\%)$ & $10,20,30$ \\
Working pressure $\left(\mathrm{P}_{\text {work }}\right)(\mathrm{mT})$ & $\mathrm{RT}$ \\
Substrate temperature $\left(\mathrm{T}_{\text {sub }}\right)\left({ }^{\circ} \mathrm{C}\right)$ & $5,15,30,60$ \\
Deposition time $\left(\mathrm{t}_{\text {dep }}\right)(\mathrm{min})$ & $200,400,500$ \\
Annealing temperature $\left(\mathrm{T}_{\text {anneal }}\right)\left({ }^{\circ} \mathrm{C}\right)$ & 3 liter $/$ min of $\mathrm{Ar}$ \\
Annealing ambient & 60 \\
\hline Annealing time $\left(\mathrm{t}_{\text {anneal }}\right)(\mathrm{min})$ & \\
\hline
\end{tabular}

The film thickness of the AZO films was investigated by wavelength variable ellipsometer. The surface morphology of the AZO films was investigated by the noncontact mode of atomic-force microscope (AFM). The structural properties of the AZO films was investigated by the $\mathrm{q}-2 \mathrm{q}$ scan of X-ray diffractometer (XRD). The electrical 
properties of the AZO films was investigated by the Hall measurement of van der Pauw type. The optical properties of the AZO films was investigated by UV-VIS spectrophotometer.

\section{Results and discussion}

Figure 1 shows the relationships between film thickness (or deposition rate) and plasma power, working gas ratio, working pressure, and deposition time, respectively, in the AZO films. The film thickness (or deposition rate) increases with the increase of plasma power, decreases with the increases of working gas ratio and working pressure, and is nearly constant with deposition time (for the case of the deposition rate). The change of the deposition rate in the AZO films fabricated by RF-sputter is ascribed to the change of sputter yield. The sputter yield has the direct relationship with the plasma power and the working pressure: the plasma power supplies the incident energy of sputtering particles, which is proportional to the sputter yield and the working pressure reduces the mean-free path of sputtering particles, which is proportional to the sputter yield [11,12]. Also, the sputter yield is related to the working gases and its ratio: the sputtering yield in the gas mixture including $\mathrm{O}_{2}$ is reported to be dropped by the three mechanisms of decrease of mass transfer rate effect of target poisoning, and increase of electron affinity [11,12]. It is no wonder that the sputter yield should be independent of the deposition time.
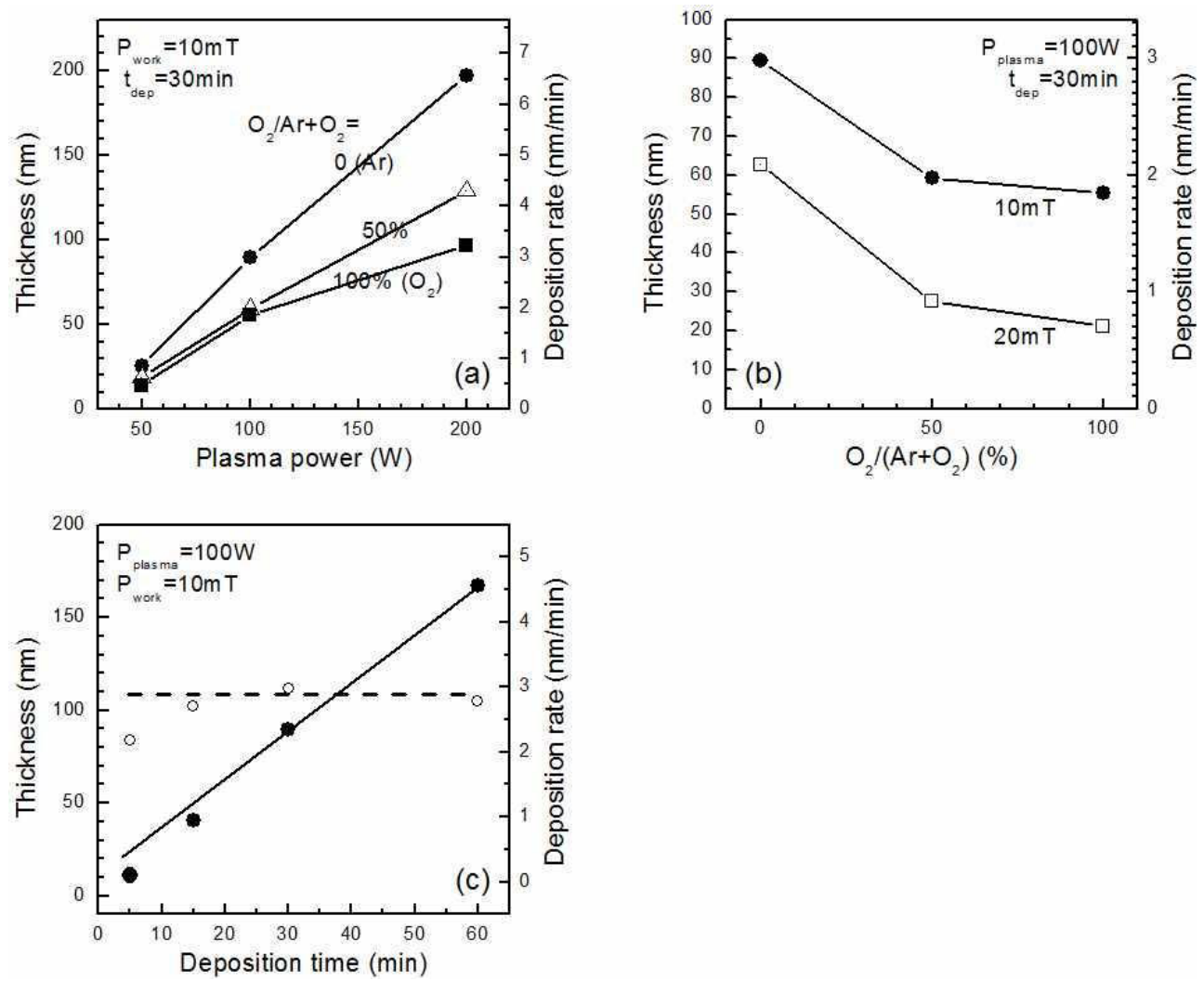

Fig. 1. Relationships between film thickness (or deposition rate) and plasma power, working gas ratio, working pressure, and deposition time, respectively, in the AZO films: (a) film thickness as a function of plasma power; (b) film thickness as a function of working gas ratio; and (c) film thickness as a function of deposition time. 

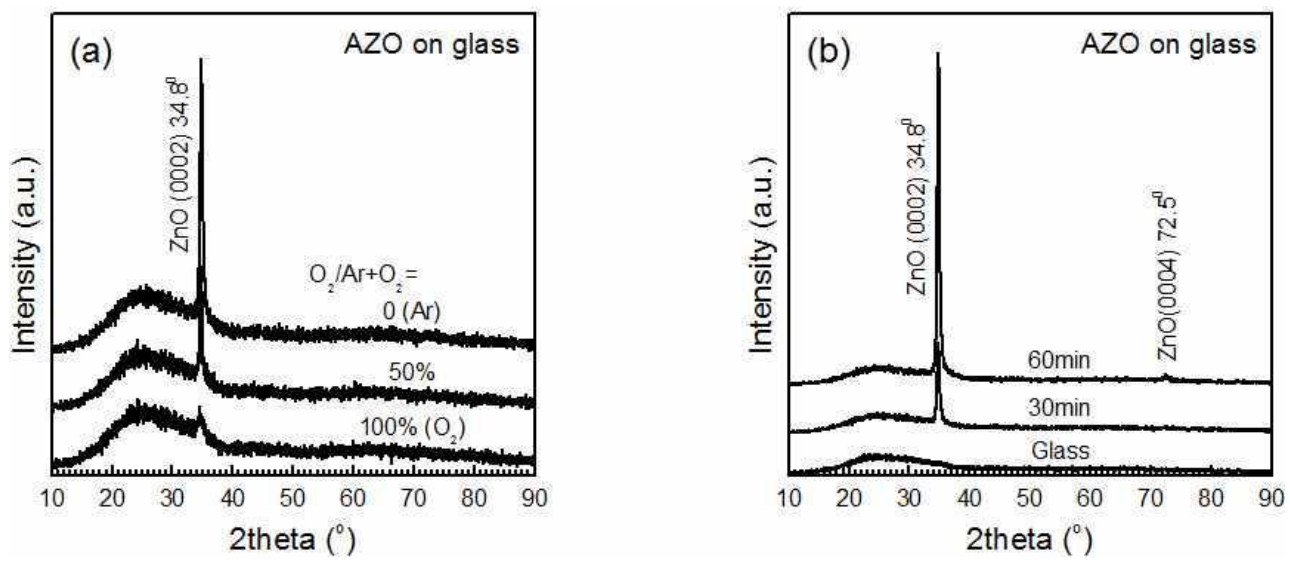

Fig. 2. Representative X-ray diffraction curves of the AZO films: (a) X-ray diffraction curve as a function of working gas ratio at a deposition time of $30 \mathrm{~min}$; and (b) X-ray diffraction curve as a function of deposition time at a working gas ratio of 0 (Ar).

Figure 2 shows the representative X-ray diffraction curves in the AZO films fabricated on glass substrates by using RF-sputter. Figs. 2(a) and 2(b) show the X-ray diffraction curves as a function of working gas ratio at a deposition time of $30 \mathrm{~min}$ and as a function of deposition time at a working gas ratio of 0 (Ar), respectively. A broad peak in the range of $10-40^{\circ}$ is assigned to the amorphous $\mathrm{SiO}_{2}$. (The bare glass substrate has the peak of the same shape at the same position.) A peak of $34.8^{\circ}$ is assigned to the (0002) planes of $\mathrm{ZnO}$ lattices $[11,12]$. The diffraction peak intensity decreases with the increase of working gas ratio, which means that $\mathrm{Ar}$ surrounding is better in the AZO film formation than $\mathrm{O}_{2}$ surrounding. The diffraction peak intensity increases with the increase of deposition time, which is due to the increase of diffracting volume by the increased film thickness. On the other hand, in the 60-min sample the peak of $\mathrm{ZnO}(0004)$ planes begins to be exposed at the position of $72.5^{\circ}$. Also, other phases related to $\mathrm{ZnO}$ and $\mathrm{AZO}$ were not observed in the wide range of $20-80^{\circ}$, which means that the AZO films deposited on glass substrates have a preferred orientation of the c-axis [11,12]. All the other AZO films had the similar X-ray diffraction curves. 

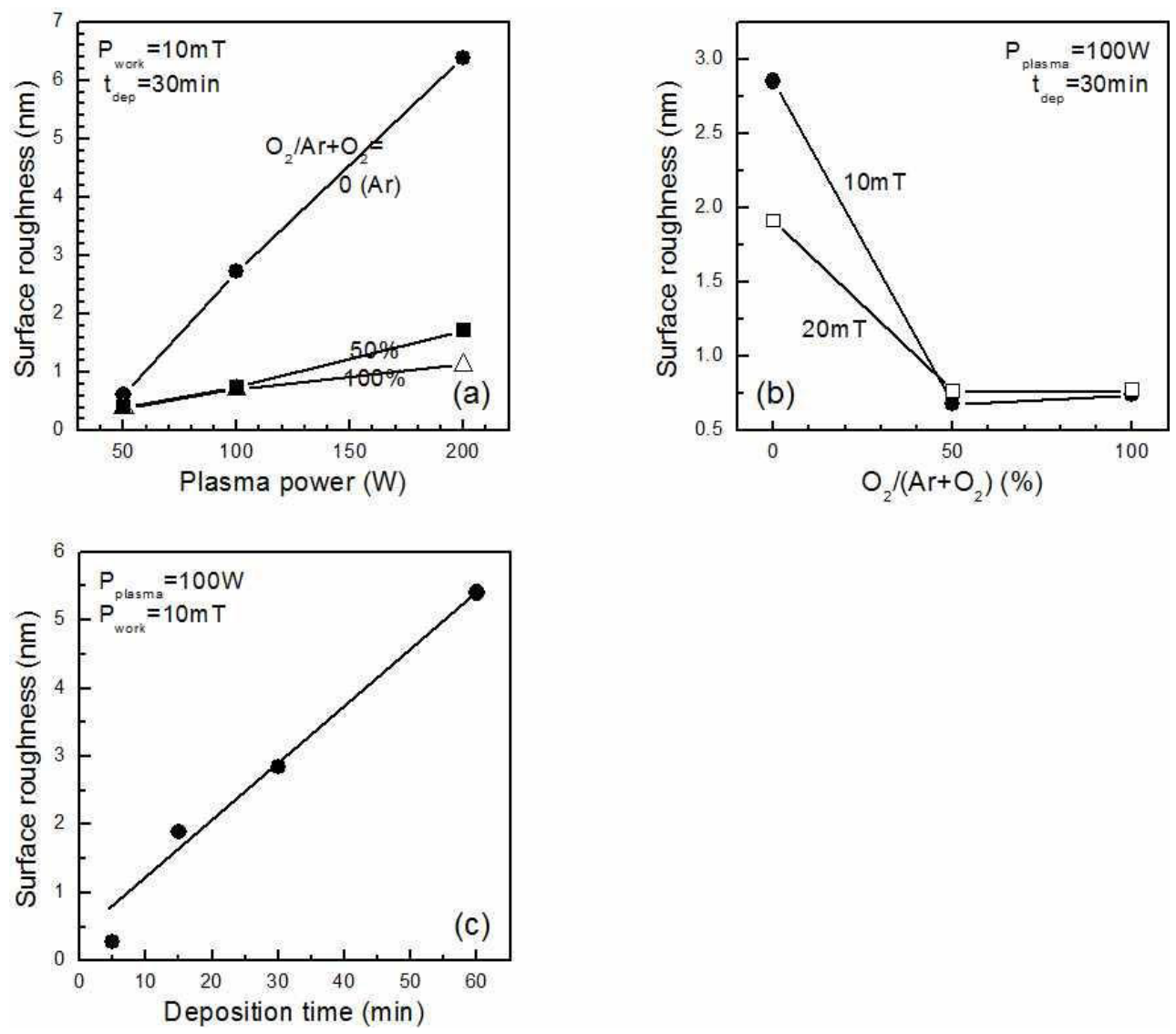

Fig. 3. Relationships between surface roughness and plasma power, working gas ratio, working pressure, and deposition time, respectively, in the AZO films: (a) surface roughness as a function of plasma power; (b) surface roughness as a function of working gas ratio; and (c) surface roughness as a function of deposition time.

Figure 3 shows the relationships between surface roughness and plasma power, working gas ratio, working pressure, and deposition time, respectively, in the AZO films. The surface roughness increases with the increase of plasma power, decreases with the increases of working gas ratio and working pressure, and increases with the increase of deposition time. These results exactly follow the relationships between film thickness and sputtering parameters in Fig. 1, which means the increase and decrease of the AZO film thickness offers the decisive factor to the increase and decrease of its surface roughness. On the other hand, Fig. 4 shows the representative surface morphologies in the AZO films fabricated on glass substrates by using RF-sputter, which is as a function of deposition time. All samples commonly have the surface structures composed of uniform nano-sized grains that mean the typical evidence of a textured growth along the c-axis $[11,12]$, which is consistent to the results of Fig. 2. The nano-sized grains grow 3-dimensionally with the increase of the deposition time, inducing the increase of the surface roughness in Fig. 3(c). All the other AZO films had the similar surface morphologies. 


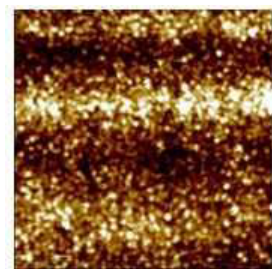

$5 \mathrm{~min}$

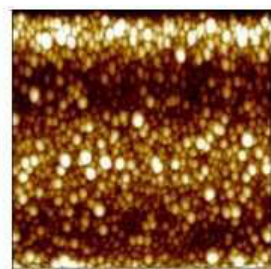

$15 \mathrm{~min}$

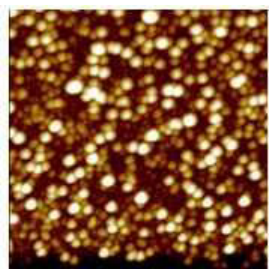

$30 \mathrm{~min}$

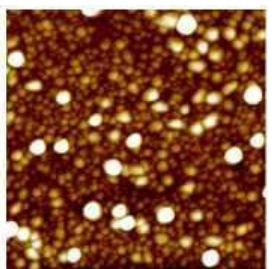

$60 \mathrm{~min}$

Fig. 4. Representative surface morphologies in the AZO films fabricated on glass substrates by using RF-sputter, which are as a function of deposition time.
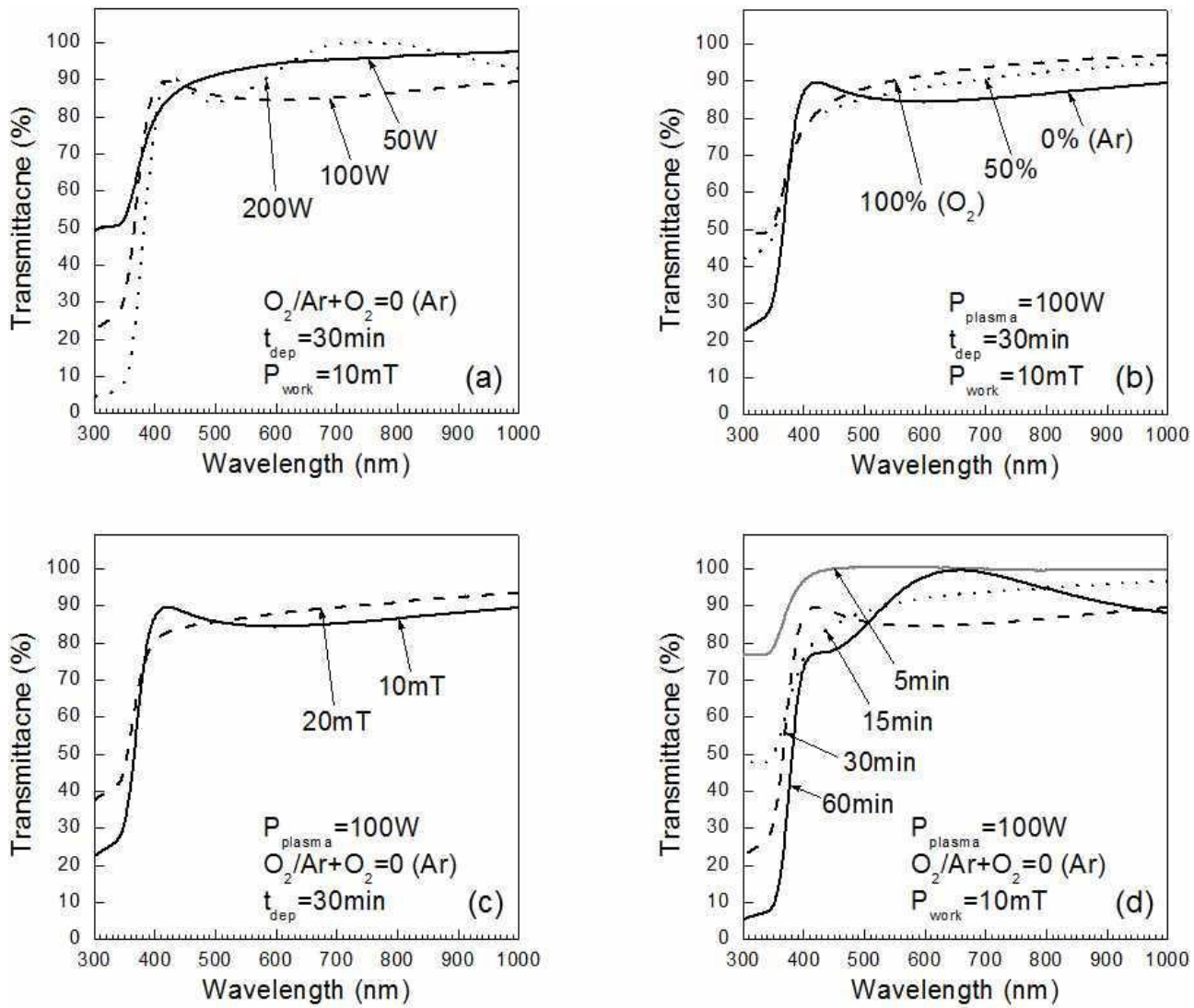

Fig. 5. Optical transmittance spectra of the AZO films as functions of (a) plasma power, (b) working gas ratio, (c) working pressure, and (d) deposition time.

Figure 5 shows the optical transmittance spectra of the AZO films as functions of plasma power, working gas ratio, working pressure, and deposition time, respectively. All samples have the high transmittance above $80 \%$ in the wide range of near UV - near IR $(400-1000 \mathrm{~nm})$, irrespective of deposition conditions. The plasma power and the deposition time relatively give a large influence on the optical transmittance, compared to the working gas ratio and the working pressure, whose origin is related to the fact that the plasma power and the deposition time more seriously change the thickness and surface roughness of the AZO films than other sputtering parameters. The change of absorption edge in the UV region of $300-350 \mathrm{~nm}$ is due not to the shift of band edge but to the thin 
film thickness smaller than extinction length. As a result, the AZO films fabricated on glass by RF-sputtering are found to have the high optical transmittance in the wide wavelength range.
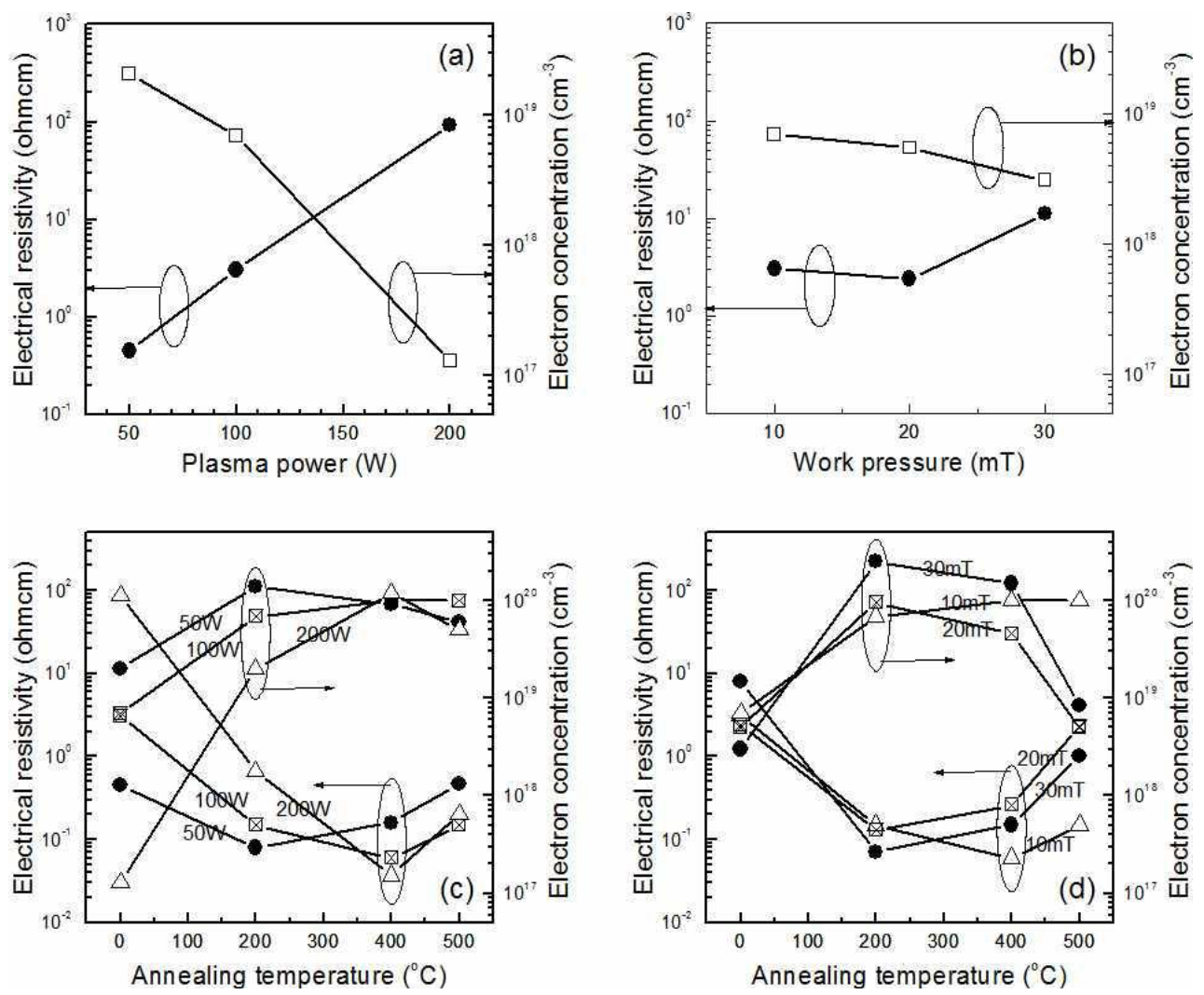

Fig. 6. Relationships between electrical resistivity (or electron concentration) and plasma power, working pressure, and annealing temperature, respectively, in the AZO films: (a) electrical resistivity and electron concentration of as-deposited AZO films as a function of plasma power, (b) electrical resistivity and electron concentration of as-deposited AZO films as a function of plasma power, (c) electrical resistivity and electron concentration as a function of annealing temperature for each plasma power, and (d) electrical resistivity and electron concentration as a function of annealing temperature for each working pressure.

Figure 6 shows the relationships between electrical resistivity (or electron concentration) and plasma power, working pressure, and annealing temperature, respectively, in the AZO films. The electrical resistivity rapidly increases with the increase of plasma power and the electron concentration is vice versa. The electrical resistivity slowly increases with the increase of working pressure and the electron concentration vice versa. Those change of electrical properties has direct relationship with the change of unintentionally generated native defects in the AZO films: the increase of the plasma power induces the increase of the native defects due to higher deposition rate, which compensate free electrons, and the increase of the working pressure induces the increase of the native defects due to shorter mean-free path, which can compensate free electrons [13,14]. Also, all AZO films have the serious dependence of the annealing temperature: an electrical resistivity of $87 \Omega \mathrm{cm}$ and an electron concentration of $1.3 \times 10^{17} \mathrm{~cm}^{-3}$ is changed to an electrical resistivity of $3.7 \times 10^{-2} \Omega \mathrm{cm}$ and an electron concentration of $1.2 \times 10^{20} \mathrm{~cm}^{-3}$ in 
terms of thermal annealing. The electrical resistivity increases with the increase of annealing temperature below a critical temperature, and above the critical temperature it decreases with the increase of annealing temperature. The electron concentration is vice versa. The break point is in the range of $200-400{ }^{\circ} \mathrm{C}$. The annealing temperature dependence of electrical properties in the AZO films is opposite to the phenomena found in high-quality single-crystalline $\mathrm{ZnO}$ films, reported previously. Those change of electrical properties by the thermal annealing has direct relationship with the formation and annihilation of the native defects: the increase of the electron concentration in the lowtemperature region is due to the annihilation of the native defects, which weaken the effect of carrier compensation, and the decrease of the electron concentration in the hightemperature region is due to the formation of the native defects, which strengthen the effect of carrier compensation $[4,5,15,16]$. On the other hand, the relationship between electrical properties and working gas ratio in the AZO films were excluded in this discussion, because the electrical resistance was significantly increased as much as any current does not flow in the AZO films fabricated under the working gases of the mixture of Ar and O2.

\section{Conclusions}

In this work, we reported on the fabrication process of AZO TCO films on glass substrates by RF-sputtering, their physical properties such as surface, structural, optical, and electrical properties, and influence of thermal annealing on the electrical properties of AZO TCO films.

\section{References}

1. K. Ellmer, A. Klein, B. Rech (Springer, Berlin, 2008)

2. P. Barquinha, R. Martins, L. Pereira, E. Fortunato, Transparent Oxide Electronics: from material to device (John Wiley \& Sons, 2012)

3. A. Facchetti, T.J. Marks, Transparent Electronics: from synthesis to applications, (John Wiley \& Sons, 2010)

4. D.C. Oh, T. Suzuki, J.J. Kim, H. Makino, T. Hanada, M.W. Cho, T. Yao, Appl. Phys. Lett., 86(3), 032909 (2005)

5. D.C. Oh, S.H. Park, H. Goto, I.H. Lim, M.N. Jung, J.H. Chang, T. Yao, J.S. Song, C.H. Bae, C.S. Han, K.W. Koo, Appl. Phys. Lett., 95(15), 151908 (2009)

6. T. Minami, Semicond Sci. Tech., 20(4), 35 (2005)

7. T. Minami, K. Oohashi, S. Takata, Thin Solid Films, 193, 721 (1990)

8. H. Agura, A. Suzuki, T. Matsushita, T. Aoki, M. Okuda, Thin Solid Films, 445(2), 263 (2003)

9. A. Hafdallah, F. Yanineb, M.S. Aida, N. Attaf, J. Alloy Compd., 509(26), 7267 (2011)

10. T.W. Nam, C.W. Lee, H.J. Kim, H.J. Kim, Appl. Surf. Sci., 295, 260 (2014)

11. K.B. Kim, S.M. Lee, D.C. Oh, H.J. Ko, J. Korean Phys. Soc., 67(4), 676 (2015)

12. S.M. Lee, K.B. Kim, H.J. Ko, D.C. Oh, J. Nanoelectron Optoe, 11(2), 250 (2016)

13. D.K. Kim, H.B. Kim, J. Alloy Compd., 509(2), 421 (2011)

14. W. Yang, Z. Wu, Z. Liu, A. Pang, Y.L. Tu, Z.C. Feng, Thin Solid Films, 519(1), 31 (2010)

15. D.C. Oh, H.J. Ko, S.K. Han, S.K. Hong, W.G. Jeong, T. Yao, Appl. Phys. Express, 5(7), 075801 (2012)

16. D.C. Oh, T.Taki, I.H. Im, S.H. Park, T. Hanada, T. Yao, J.S. Song, J.H. Chang, M. Makino, C.S. Han, K.H. Koo, J. Vac. Sci. Technol. A, 26(2), 259 (2008) 